\title{
Integrating advanced discrete choice models in mixed integer linear optimization
}

\author{
Meritxell Pacheco \\ Shadi Sharif Azadeh, Michel Bierlaire, Bernard Gendron
}

Transport and Mobility Laboratory (TRANSP-OR)

École Polytechnique Fédérale de Lausanne

February, 2018 


\section{Outline}

(1) Introduction

(2) Choice model

(3) Optimization model

(4) Demand-based benefit maximization

(5) Case study

(6) Conclusions and ongoing work 


\section{Introduction}

\section{(1) Introduction}

\section{(2) Choice model}

(3) Optimization model

(4) Demand-based benefit maximization

(5) Case study

(6) Conclusions and ongoing work 


\section{Discrete choice models}

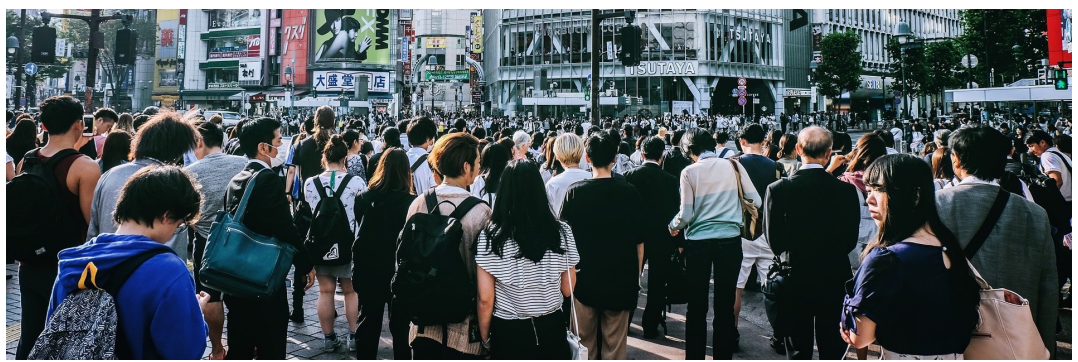

- Demand modeling

- Disaggregate level
- Heterogeneity of the population

- Predict the choice 


\section{Why are they not in OR?}

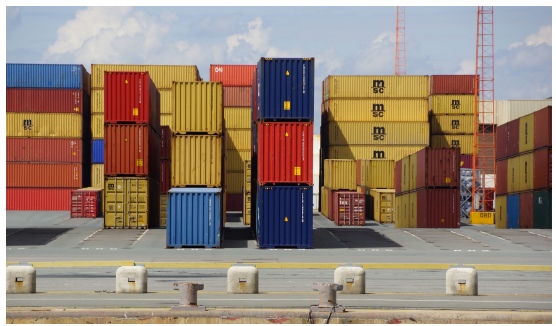

- Tractability

- Linearity and/or convexity

- MILP models 


\section{Why are they not in OR?}

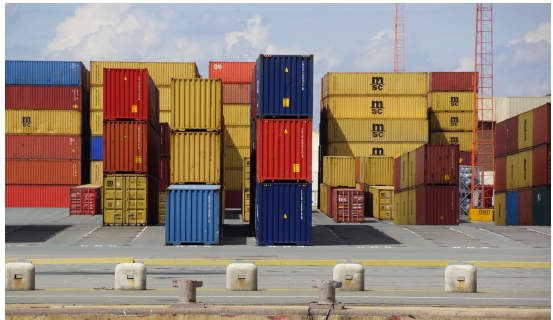

- Tractability

- Linearity and/or convexity

- MILP models

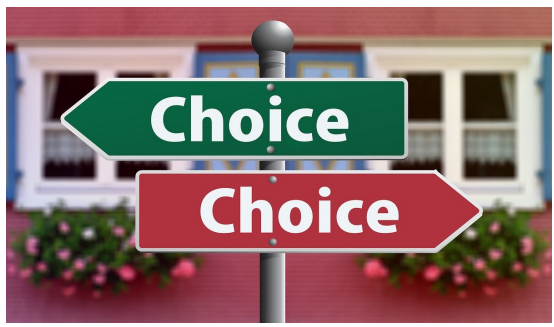

- Behavioral realism

- Unrealistic assumptions

- Complex formulations 


\section{Bridging the gap}

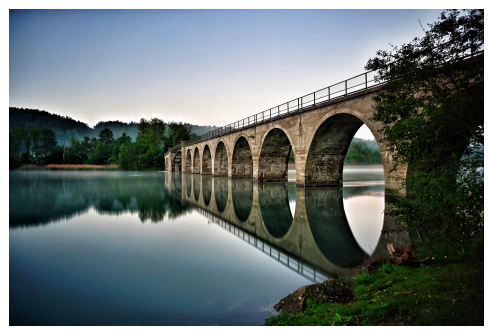

- General framework integrating

- choice model (demand)

- MILP model (supply)

- Simulation to linearize the choice model 


\section{General framework}

- Exogenous variables explaining the choice: $x^{d} \in \mathbb{R}^{D}$ 


\section{General framework}

- Exogenous variables explaining the choice: $x^{d} \in \mathbb{R}^{D}$

- Exogenous variables involved in the optimization model: $x^{s} \in \mathbb{R}^{S}$ 


\section{General framework}

- Exogenous variables explaining the choice: $x^{d} \in \mathbb{R}^{D}$

- Exogenous variables involved in the optimization model: $x^{s} \in \mathbb{R}^{S}$

- Endogenous variables appearing in both models: $x^{e} \in \mathbb{R}^{E}$

- Characterize the interactions (e.g.: price)

- $\ell^{e} \leq x^{e} \leq m^{e}$ 


\section{(2) Choice model}

(3) Optimization model

(4) Demand-based benefit maximization

(5) Case study

(6) Conclusions and ongoing work 


\section{Choice set and population}

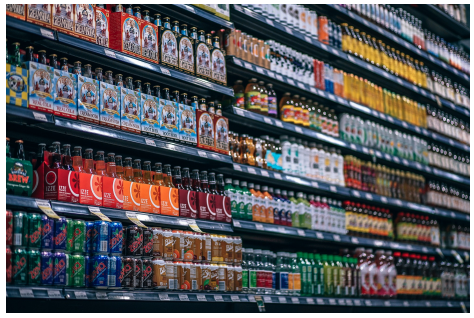

- Set of alternatives $\mathcal{C}(i)$

- Capacity: $c_{i} \geq 1$

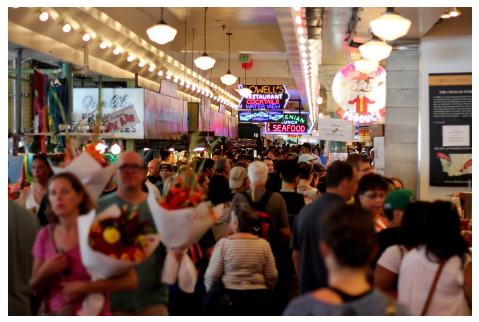

- $N$ individuals $(n)$

- Individual choice set $\mathcal{C}_{n} \subseteq \mathcal{C}$ 


\section{Choice set and population}

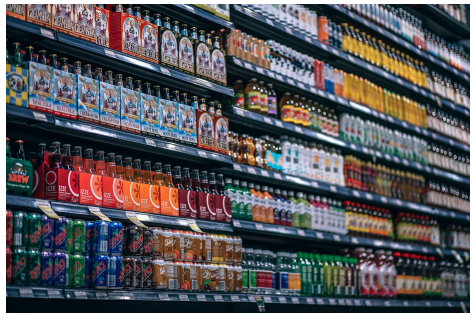

- Set of alternatives $\mathcal{C}(i)$

- Capacity: $c_{i} \geq 1$

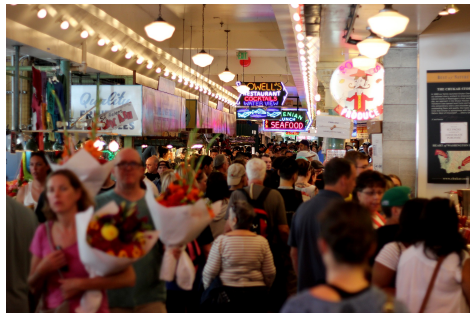

- $N$ individuals $(n)$

- Individual choice set $\mathcal{C}_{n} \subseteq \mathcal{C}$

$$
y_{i n}= \begin{cases}1 & \text { if alternative } i \text { is offered to } n \\ 0 & \text { otherwise }\end{cases}
$$




\section{Utility function and behavioral assumption}

$$
U_{i n}\left(x^{d}, x^{e} ; \varepsilon_{i n}\right)=V_{i n}\left(x^{d}, x^{e}\right)+\varepsilon_{i n}
$$

- Utility function

- Deterministic part: $V_{i n}\left(x^{d}, x^{e}\right)=\sum_{k} \beta_{k} x_{i n k}^{e}+g^{d}\left(x^{d}\right)$

- Random term: $\varepsilon_{\text {in }}$ 


\section{Utility function and behavioral assumption}

$$
U_{i n}\left(x^{d}, x^{e} ; \varepsilon_{i n}\right)=V_{i n}\left(x^{d}, x^{e}\right)+\varepsilon_{i n}
$$

- Utility function

- Deterministic part: $V_{i n}\left(x^{d}, x^{e}\right)=\sum_{k} \beta_{k} x_{i n k}^{e}+g^{d}\left(x^{d}\right)$

- Random term: $\varepsilon_{\text {in }}$

- Behavioral assumption

- $i$ chosen by $n$ if $U_{\text {in }} \geq U_{j n}, \forall j \in \mathcal{C}_{n}$

- $P_{n}\left(i \mid x^{d}, x^{e}\right)=\operatorname{Pr}\left(U_{i n} \geq U_{j n}, \forall j \in \mathcal{C}_{n}\right)$ 


\section{Simulation}

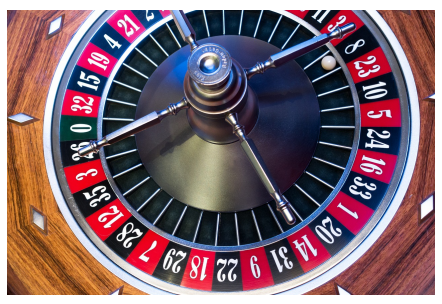

- $R$ draws from the distribution of $\varepsilon_{\text {in }}$

- $\xi_{\text {in } 1}, \ldots, \xi_{\text {in } R}$

- Behavioral scenario

$$
U_{i n r}=\sum_{k} \beta_{k} x_{i n k}^{e}+g^{d}\left(x^{d}\right)+\xi_{i n r}
$$




\section{Availability}

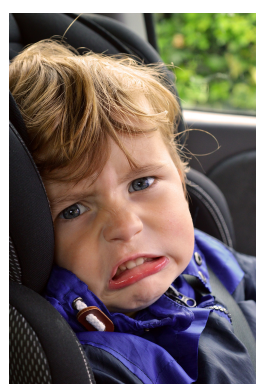

- Not considered

- $y_{\text {in }}=0 \forall i \notin \mathcal{C}_{n}$ 


\section{Availability}

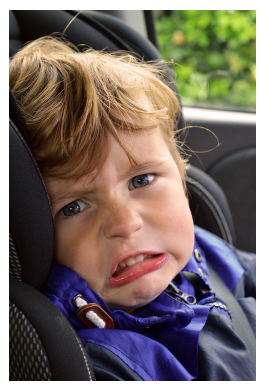

- Not considered

- $y_{\text {in }}=0 \forall i \notin \mathcal{C}_{n}$

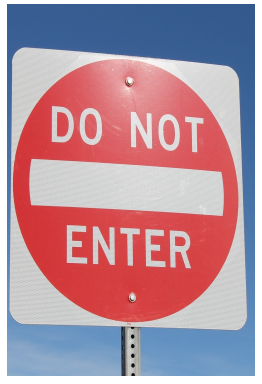

- Not offered

- Model's decision 


\section{Availability}

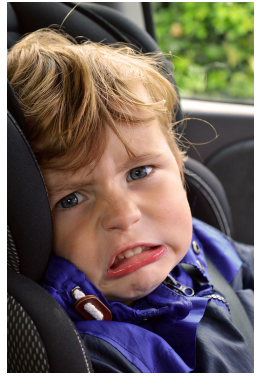

- Not considered

- $y_{\text {in }}=0 \forall i \notin \mathcal{C}_{n}$

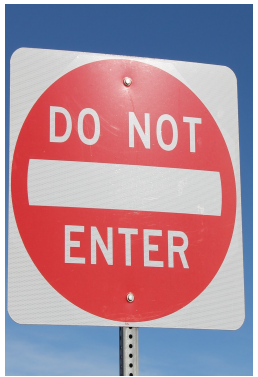

- Not offered

- Model's decision

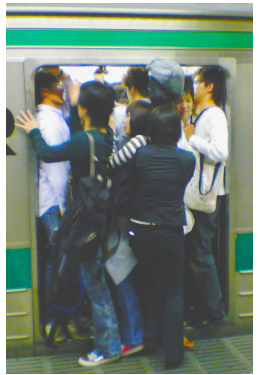

- Reached capacity

- $\boldsymbol{y}_{\text {inr }} \leq y_{\text {in }}$ 


\section{Discounted utility}

$$
\begin{gathered}
z_{i n r}= \begin{cases}U_{i n r} & \text { if } y_{i n r}=1 \\
\ell_{n r} & \text { if } y_{i n r}=0\end{cases} \\
\ell_{n r} \leq z_{i n r} \\
z_{i n r} \leq \ell_{n r}+M_{i n r} y_{i n r} \\
U_{i n r}-M_{i n r}\left(1-y_{i n r}\right) \leq z_{i n r} \\
z_{i n r} \leq U_{i n r}
\end{gathered}
$$




\section{Choice}

$$
U_{n r}=\max _{i \in \mathcal{C}} z_{i n r}
$$

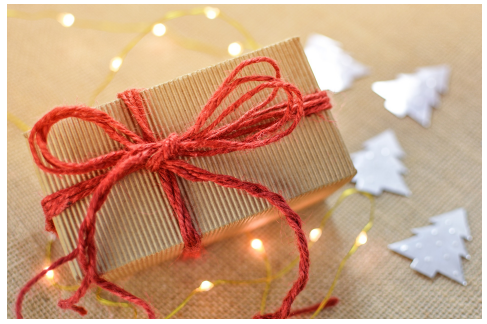

$$
\begin{aligned}
z_{i n r} & \leq U_{n r} \\
U_{n r} & \leq z_{i n r}+M_{n r}\left(1-w_{i n r}\right) \\
w_{i n r} & \leq y_{i n r} \\
\sum_{i \in \mathcal{C}} w_{i n r} & =1
\end{aligned}
$$




\section{Expected demand}

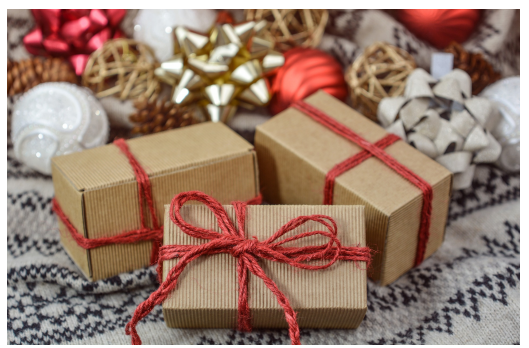

$$
D_{i}=\frac{1}{R} \sum_{r=1}^{R} \sum_{n=1}^{N} w_{i n r}
$$




\section{Capacity allocation: priority list}

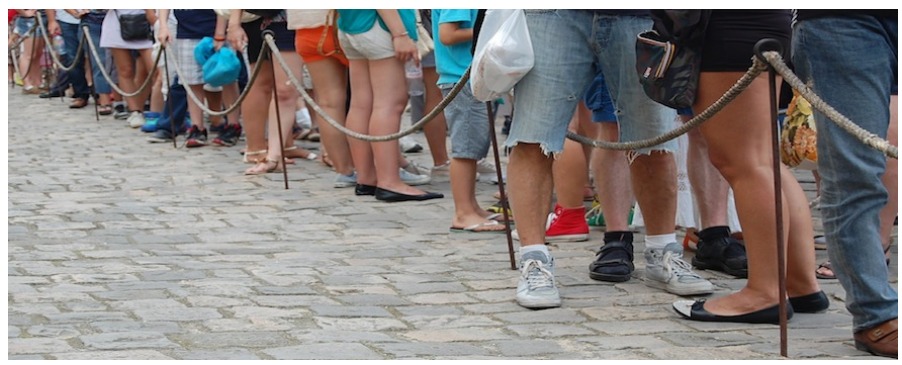

- Exogenous to the model

- Relationship with individuals
- Numbering of individuals

- Example: random arrival 


\section{Capacity allocation: capacity cannot be exceeded}

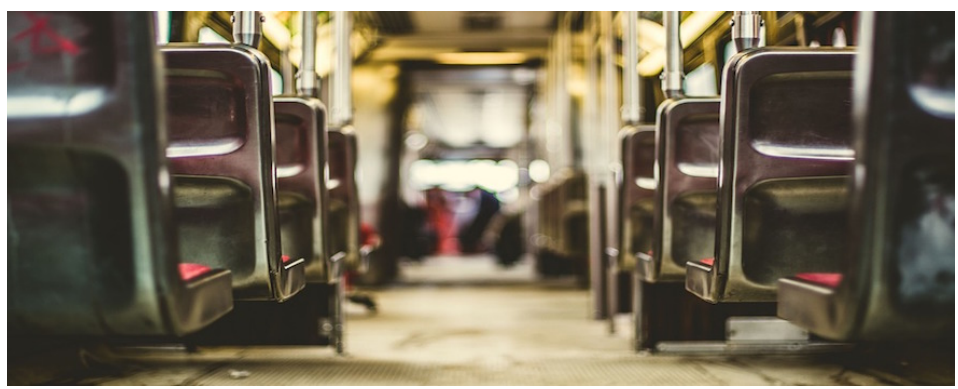

$$
\sum_{m=1}^{n-1} w_{i m r} \leq\left(c_{i}-1\right) y_{i n r}+(n-1)\left(1-y_{i n r}\right)
$$




\section{Capacity allocation: capacity has been reached}

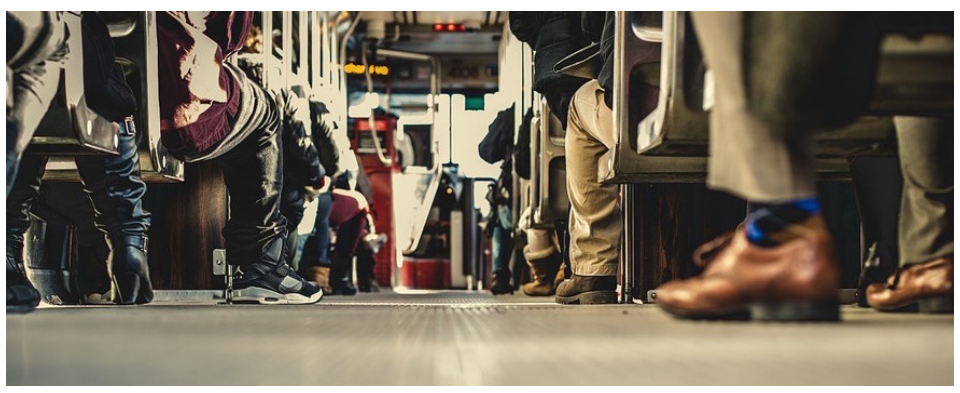

$$
c_{i}\left(y_{i n}-y_{i n r}\right) \leq \sum_{m=1}^{n-1} w_{i m r}
$$


(1) Introduction

(2) Choice model

(3) Optimization model

(4) Demand-based benefit maximization

(5) Case study

(6) Conclusions and ongoing work 


\section{General optimization model (MILP)}

$g^{s}\left(x^{s}, x^{e}\right)$

$$
\begin{aligned}
h^{s}\left(x^{s}, x^{e}\right) & =0 \\
\ell^{e} \leq x^{e} & \leq m^{e} \\
x_{z}^{e} & \in \mathbb{Z}
\end{aligned}
$$

- Objective function (linear)

- Relates decisions at an aggregate level

- Set of constraints (linear)

- Feasible configuration of the variables 


\section{Applications}

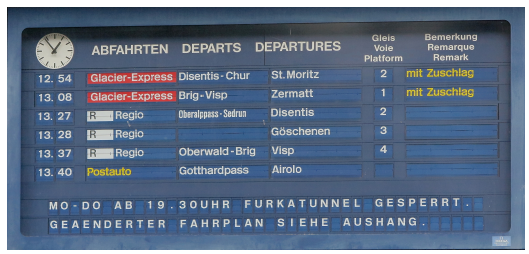

- Design of a train timetable

- Objective: maximize profit

- Constraints: passenger satisfaction

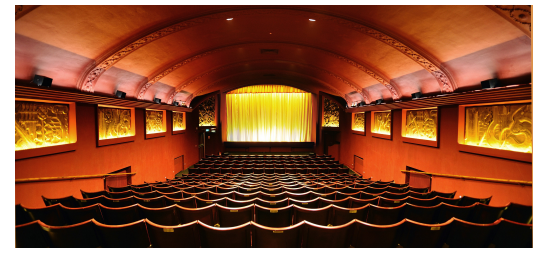

- Schedule, what movie...

- Objective: total benefit

- Constraints: one movie per theater, capacity 


\section{Demand-based benefit maximization}

(2) Choice model

(3) Optimization model

(4) Demand-based benefit maximization

(5) Case study

(6) Conclusions and ongoing work 


\section{General setting}

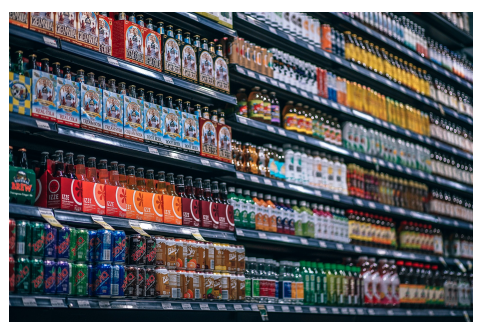

- Set of services $\mathcal{C}(i)$

- Capacity: $c_{i} \geq 1$

- Opt-out option $(i=0)$

- Maximize benefit

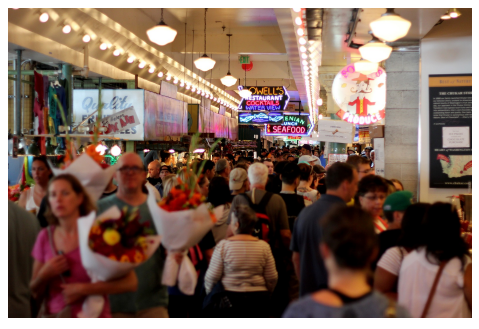

- $N$ customers $(n)$

- Individual choice set $\mathcal{C}_{n} \subseteq \mathcal{C}$

- $0 \in \mathcal{C}_{n} \forall n$

- Price to pay: $p_{i n}$ 


\section{Benefit maximization}

$$
\begin{array}{cc}
\max \sum_{i>0}\left(G_{i}-C_{i}\right) & \\
G_{i}=\frac{1}{R} \sum_{n=1}^{N} \sum_{r=1}^{R} p_{i n} w_{i n r} & C_{i}=\left(f_{i}+v_{i} c_{i}\right) y_{i}
\end{array}
$$

- Product of variables

- Binary representation of price

- $\frac{1}{10^{k}} a_{i n} \leq p_{\text {in }} \leq \frac{1}{10^{k}} b_{\text {in }}$

- $L_{\text {in }}=\left\lceil\log _{2}\left(b_{i n}-a_{i n}+1\right)\right\rceil$ variables
- $f_{i}$ fixed cost

- $v_{i}$ variable cost

- $y_{i}=1$ if $i$ is offered $\forall n$

- $y_{i n}=y_{i} \forall n$ 


\section{Price characterization}

$$
\begin{gathered}
p_{i n}=\frac{1}{10^{k}}\left(a_{i n}+\sum_{\ell=0}^{L_{i n}-1} 2^{\ell} \lambda_{i n \ell}\right) \\
G_{i}=\frac{1}{R} \frac{1}{10^{k}} \sum_{n=1}^{N} \sum_{r=1}^{R}\left(a_{i n}+\sum_{\ell=0}^{L_{i n}-1} 2^{\ell} \lambda_{i n \ell}\right) w_{i n r} \\
=\frac{1}{R} \frac{1}{10^{k}}\left[\sum_{n=1}^{N} \sum_{r=1}^{R}\left(a_{i n} w_{i n r}+\sum_{\ell=0}^{L_{i n}-1} 2^{\ell} \alpha_{i n r \ell}\right)\right] \\
\text { where } \alpha_{i n r \ell}=\lambda_{\text {in } \ell} w_{i n r}
\end{gathered}
$$




\title{
Resulting MILP
}

\author{
max benefit \\ subject to availability \\ utility definition \\ discounted utility \\ choice \\ capacity allocation \\ price selection
}




\section{(2) Choice model}

(3) Optimization model

(4) Demand-based benefit maximization

(5) Case study

(6) Conclusions and ongoing work 


\section{Parking choices}
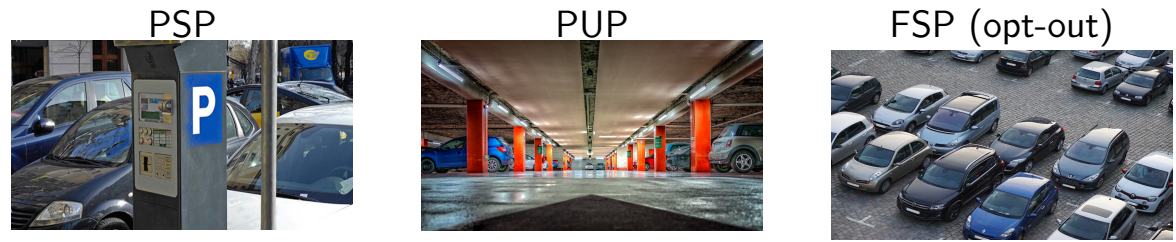

- $N=50$ customers

- Mixtures of a logit model

- $\mathcal{C}=\{$ PSP, PUP, FSP $\}$

- $y_{i n}=y_{i} \quad \forall n$

- $\mathcal{C}_{n}=\mathcal{C} \quad \forall n$

- $p_{\text {in }}=p_{i} \quad \forall n$ 


\section{General experiments}

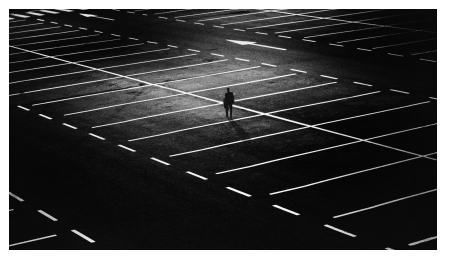

- Maximization of revenue

- Unlimited capacity

- Capacity of 20 spots for PSP and PUP

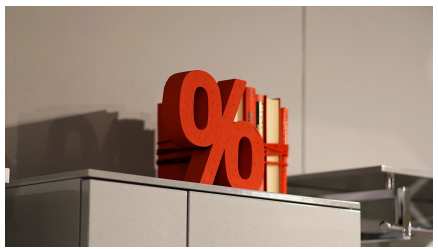

- Reduced price for residents

- Two scenarios

(1) Subsidy offered by the municipality

(2) Operator is obliged to offer a reduced price 


\section{Uncapacitated vs Capacitated case (1)}

\section{Uncapacitated}

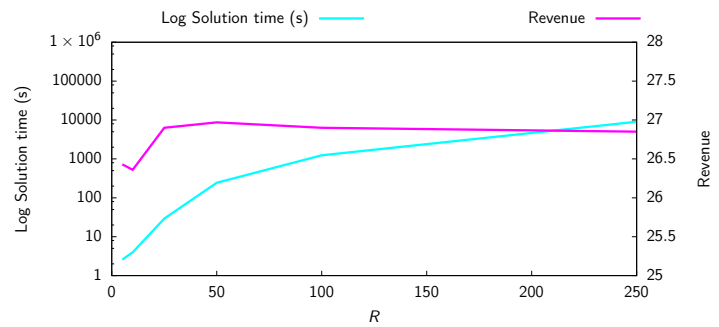

\section{Capacitated}

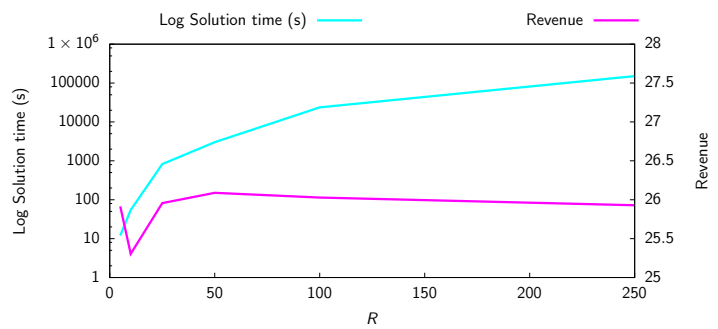




\section{Uncapacitated vs Capacitated case (2)}

\section{Uncapacitated}

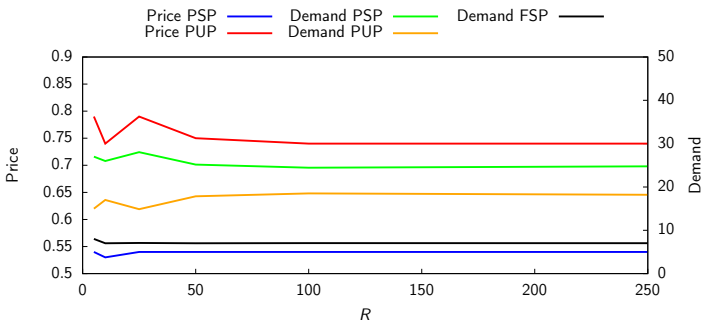

\section{Capacitated}

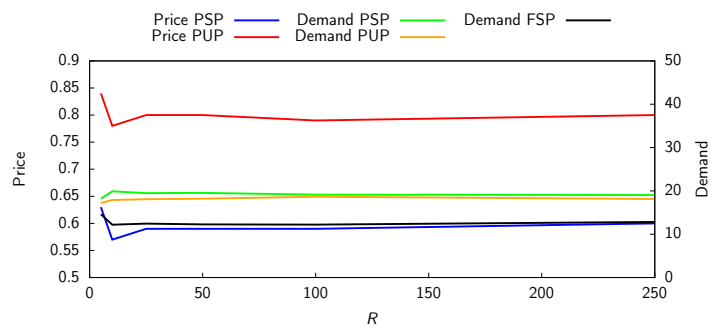




\section{Price differentiation by population segmentation}

Subsidy offered by the municipality

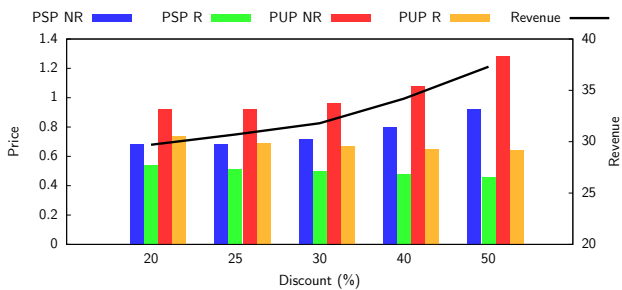

Operator is obliged to offer a reduced price

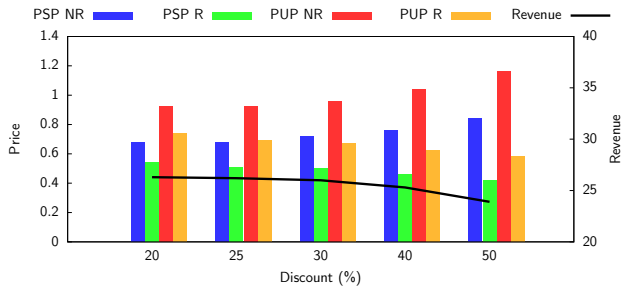




\section{(1) Introduction}

(2) Choice model

(3) Optimization model

(4) Demand-based benefit maximization

(5) Case study

(6) Conclusions and ongoing work 


\section{Conclusions and ongoing work}

- Powerful tool to configure systems based on heterogenous behavior

- Computationally expensive

- Decomposition techniques $\Rightarrow$ Lagrangian relaxation

- Operator subproblem: FLP

- Customer subproblem: iterative method (customers) 


\section{Questions?}

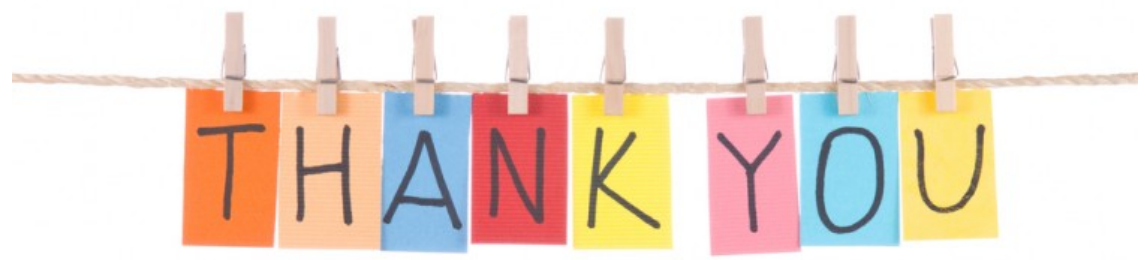

meritxell.pacheco@epfl.ch 